\title{
Osteoconductive Lattice Microarchitecture for Optimized Bone Regeneration
}

\author{
Michael De Wild, ${ }^{1}$ Chafik Ghayor, Simon Zimmermann,, Jasmine Rüegg,, Flora Nicholls, \\ Felix Schuler, Tse-Hsiang Chen, and Franz E. Weber ${ }^{2,4,5}$
}

\begin{abstract}
Selective laser melting (SLM) is one methodology to realize additive manufacturing and is mainly used to join metal powder in a layer-by-layer manner to produce a solid three-dimensional (3D) object. For bone tissue engineering purposes, scaffolds can readily be designed as 3D data model and realized with titanium known for its excellent osseointegration behavior. The microarchitecture, that is, design with submillimeter features, of additively manufactured scaffolds is in many cases a lattice structure. This study aimed to apply SLM that allows a high degree of microarchitectural freedom to generate lattice structures and to determine the optimal distance between rods and the optimal diameter of rods for osteoconduction (bone ingrowth into scaffolds) and bone regeneration. For the biological readout, diverse SLM-fabricated titanium implants were placed in the calvarium of rabbits and new bone formation and defect bridging were determined after 4 weeks of healing. The results from the middle section of the defects show that with a lattice microarchitecture, the optimal distance between titanium rods is around $0.8 \mathrm{~mm}$ and the optimal rod dimension is between 0.3 and $0.4 \mathrm{~mm}$ to optimize defect bridging and bone regeneration.
\end{abstract}

Keywords: selective laser melting, titanium, bone regeneration, bone repair, osteoconduction, grid architecture, lattice architecture, additive manufacturing

\section{Introduction}

BONE REPLACEMENT MATERIALS need to be accepted by the body and should clinically be infiltrated with bone tissue within a short time, so ideally they are osteoconductive. Bone tissue engineering of osteoconductive biomaterials like other tissue engineering approaches normally relies on the combination of cells, bioactive factors, and biomaterial scaffold to facilitate and accelerate the regeneration of bone tissue. ${ }^{1,2}$ The macroarchitecture defined as the millimeter-to-centimetersized outer shape of the scaffold is in most cases identical to the dimensions of the bone defect and, therefore, patient spe- cific. It can be realized by several different approaches such as in situ forming implants ${ }^{3}$ or in situ forming hydrogels. ${ }^{4,5}$ If mechanically more stable personalized bone tissue scaffolds are mandatory, additive manufacturing (AM) provides the tools to produce patient-specific implants from a variety of materials including calcium phosphates and titanium. ${ }^{6,7}$

For personalized bone substitutes, the AM process could be based on converted computer tomography data from the patients or any other three-dimensional (3D) model data set. ${ }^{8}$ The process itself makes the scaffold layer upon layer, dependent on the material used by diverse methodologies, including stereolithography, selective laser sintering, three-dimensional

\footnotetext{
${ }^{1}$ School of Life Sciences, Institute for Medical and Analytical Technologies, University of Applied Sciences Northwestern Switzerland, Muttenz, Switzerland.

${ }^{2}$ Oral Biotechnology and Bioengineering, Center of Dental Medicine, University of Zurich, Zurich, Switzerland.

${ }^{3}$ Division of Surgical Research, Centre for Clinical Research University Hospital Zurich, Switzerland.

${ }^{4}$ CABMM, Center for Applied Biotechnology and Molecular Medicine, University of Zurich, Zurich, Switzerland.

${ }^{5}$ Zurich Center for Integrative Human Physiology (ZIHP), University of Zurich, Zurich, Switzerland.

(C) Michael De Wild et al. 2018; Published by Mary Ann Liebert, Inc. This Open Access article is distributed under the terms of the Creative Commons License (http://creativecommons.org/licenses/by/4.0), which permits unrestricted use, distribution, and reproduction in any medium, provided the original work is properly cited.
} 
printing (3DP), and fused deposition modeling. ${ }^{9}$ In stereolithography, ultraviolet light or laser polymerizes defined areas of individual layers to form an object from layers of photosensitive polymers. This technique was already established in $1981 .{ }^{10}$ In 1989 , selective laser sintering was developed and patented ${ }^{11}$ wherein a high-intensity laser beam is used to build an object in a metal, polymer, ceramic, or hybrid powder bed. In 3DP, developed in 1990 at the Massachusetts Institute of Technology, the object forms in a powder bed by the deposition of a liquid binder material using inkjet heads. ${ }^{12}$ One of the first studies with 3D-printed hydroxyapatite scaffolds showed deep cell proliferation into structures with dimensions and pore sizes of about $500 \mu \mathrm{m} .{ }^{13}$ Melt-extrusion AM was established in $1992^{14}$ and solution/slurry extrusion in $2002{ }^{15,16}$ In both cases, a polymeric filament is extruded through a nozzle and deposited layer-by-layer on a building platform. If applied for bone tissue engineering, extrusionbased AM mainly produces lattice structures as submillimetersized microarchitecture of the newly formed bone substitute, wherein the thickness of the deposited filament defines the minimal structure dimension. Fused deposition modeling was first explored with poly-e-caprolactone (PCL) and PCLhydroxyapatite composites by Hutmacher et al. ${ }^{17}$ and applied in humans to cover cranial defects. ${ }^{18}$ More recently, extrusion was also used to produce scaffolds from bioglass to generate grid-like structures for the treatment of large cranial defects. ${ }^{19}$

The ideal microstructure of a bone tissue engineering construct was defined in the 1990s, when porous ceramicbased bone substitutes were produced mainly with porogens, following a space-holder methodology. At that time, it was established that the pores in a bone tissue engineering scaffold are ideally between 0.3 and $0.5 \mathrm{~mm}$ in diameter. ${ }^{20-22}$ More recent work by others on porogen-based porous bone substitutes $^{23}$ and us on additively manufactured open porous scaffolds ${ }^{24,25}$ showed that bone tissue engineering scaffolds with pores exceeding $500 \mu \mathrm{m}$ are efficient for bone ingrowth and bone regeneration in vivo.

Dependent on the methodology and the materials, AM can be used to realize a wide variety of microstructures including also functionally graded scaffolds. ${ }^{26}$ Not only the macro- and microporosity of PCL scaffolds produced by a 3D plotting technique ${ }^{27}$ but also the macroscopic arrangement of the porous arrangement in ceramic scaffolds is important. ${ }^{28}$ As known from regenerative therapies by tissue engineering, the ideal pore size of scaffolds made of ceramics, synthetic polymers, and natural polymers also depends on the applied cell type ${ }^{29,30}$ in particular when studying on the molecular level ${ }^{31}$ or in vitro. ${ }^{32}$

Titanium and titanium alloys are suitable materials for bone substitutes, since they exhibit a high specific strength and support osteoblast colonization and differentiation into mature bone cells. ${ }^{33}$ Possible applications of additively manufactured titanium implants in craniomaxillofacial surgery (reviewed in Ref. $^{34}$ ) range from personalized titanium implants for the reconstruction of the orbital floor, ${ }^{35}$ the ramus mandibulae, and the condyle ${ }^{36}$ to dental implants. ${ }^{37} \mathrm{In}$ orthopedics, additively manufactured titanium constructs proved useful for long-bone defects. ${ }^{38,39}$ By AM, a mechanobiologically optimized 3D titanium-mesh structure could be realized to promote the healing of a critical-size long-bone defect in sheep by a design that reduced stress shielding. ${ }^{39}$ Ti-6Al-4V-based personalized plates were used successfully to treat complex pelvic fractures. ${ }^{40}$ In vitro experiments indicate that trabecular bone-like 3D structures produced by selective laser melting (SLM) of Ti-6Al-4V favor osteogenesis, especially at high porosity. ${ }^{41}$ Honeycomb structures with effective pore diameter from 0.2 to $0.7 \mathrm{~mm}$ produced by the same material through direct laser forming showed good cell compatibility. ${ }^{42}$ The same applies to a rapid prototyped porous nickel-titanium scaffold. ${ }^{43} \mathrm{~A}$ more extended study on pore geometry and its effect on human periosteum-derived cells was performed with diverse selective laser-melted Ti-6Al-4V bone scaffolds to show that cell proliferation depends on pore size but not on pore shape. Differentiation, however, depends on both parameters. ${ }^{44}$

Despite all these in vitro and in vivo studies, the knowledge about the effect of diverse lattice microarchitectures on the in vivo ingrowth velocity of bone tissue into the scaffold and bone formation is limited. Therefore, we used SLM of titanium $^{45}$ to produce lattice implants of systematically varied rod distances (four different porous channel widths $w$ between 300 and $1800 \mu \mathrm{m}$ ) and rod calibers (five different calibers $s$ between 200 to $1500 \mu \mathrm{m}$ ) to study the influence of those microarchitectural parameters on bone regeneration and defect bridging in a calvarial defect model system in rabbits in great detail.

\section{Materials and Methods}

\section{Implant design}

The implants were constructed with Solidworks CAD system (Solidworks V.2013 Software, www.solidworks.com, Dassault Systèmes, France). The outer macrogeometry of the implants is designed as stepped cylinders of $7.5 \mathrm{~mm}$, respectively, $6 \mathrm{~mm}$ diameter and a height of $4.2 \mathrm{~mm}$. The inner microarchitecture is constructed by cutting out symmetrically arranged square channels in all three orthogonal directions, see Figure 1. Based on most promising scaffold parameters from the literature, ${ }^{29}$ the width of the channels $w$ (distance between the rods) and the wall thickness $s$ (rod caliber) of the remaining trusses have been systematically varied in a way that complete layers of cubic unit cells are formed along the cylindrical main axis (see Fig. 1 for an overview of the designs and Table 1 for the structural values). Finally, a $0.2 \mathrm{~mm}$ fine ring completes the outermost margin of the framework to close the last layer and prevent sharp edges during surgery. Three series of implant design have been constructed:

(1) First series with constant rod calibers $s$ of $0.2 \mathrm{~mm}$, but with varied channel width $w$, that is, distance between the rods of $0.3,0.466,0.8$, and $1.8 \mathrm{~mm}$.

(2) Second series with fixed channel width $w$ of $0.5 \mathrm{~mm}$, however, with different rod calibers $s$ of $0.2,0.5$, and $1.5 \mathrm{~mm}$.

(3) Third series with fixed channel width $w$ of $0.8 \mathrm{~mm}$, however, with different rod calibers $s$ of $0.2,0.3$, and $0.4 \mathrm{~mm}$.

These geometrical values are determined to guarantee a total height of the scaffold of exact $4.2 \mathrm{~mm}$ for series 1 and 2 and uniformly distributed channels and rods with biologically meaningful profiles. To compare the individual scaffolds, 


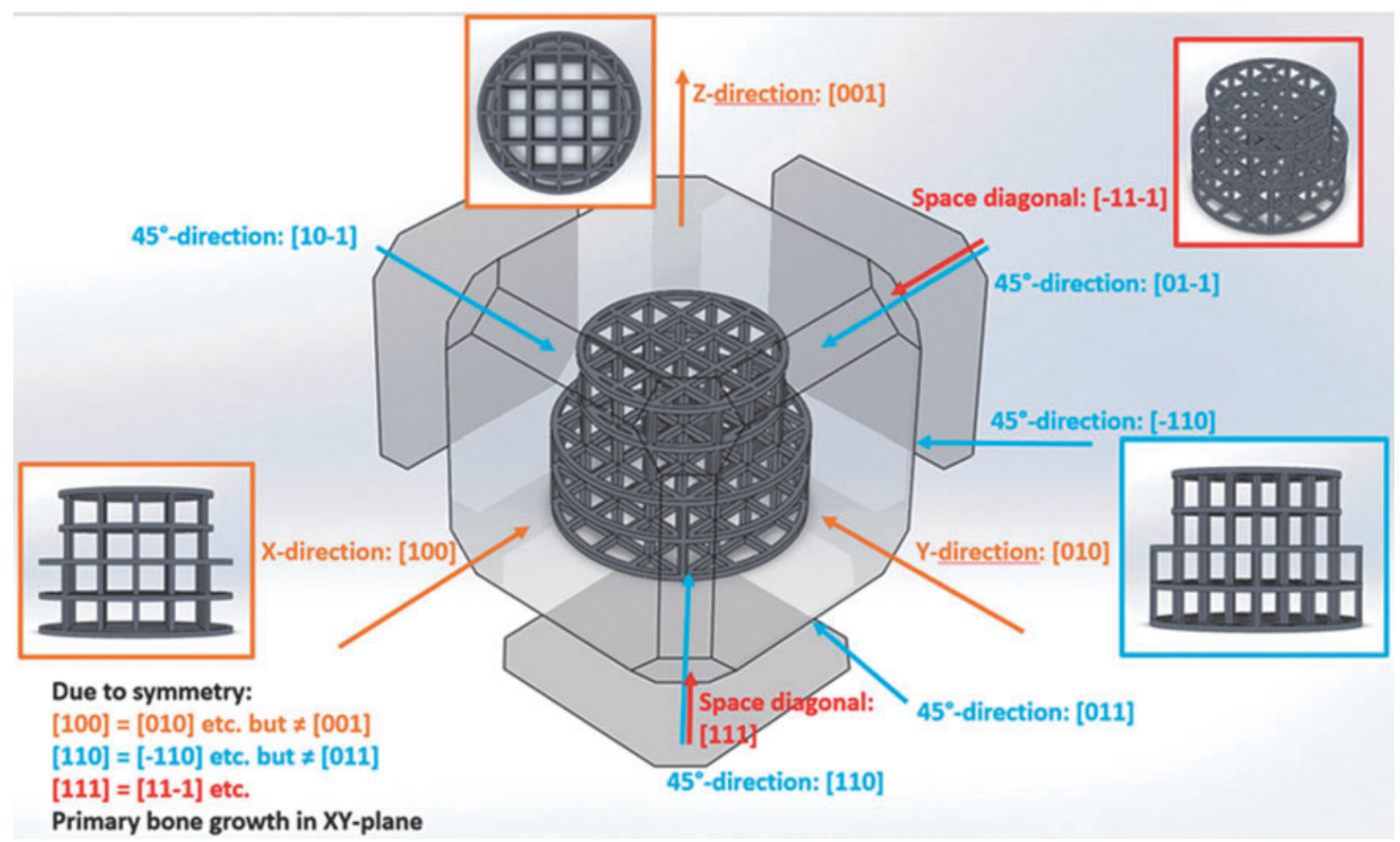

FIG. 1. Design of the tested implants. The different profile views used for characterization are shown.

their theoretical characteristics were calculated. The most important being (Table 1) the following:

- The relative free volume $\left(\mathrm{V}_{\text {free }}\right)$ describing the ratio of the free volume inside the scaffold $\left(\mathrm{V}_{\text {enclosed }}\right)$.

- The relative transparencies (relative free area in the projection of the scaffold in the different spatial directions, as shown in Fig. 1).

- The total scaffold surface, which is significant for the corrosion process.

\section{Implant production}

The implants were produced by the Realizer $250^{\mathrm{HT}}$ selective laser melting system (SLM Solutions, Lübeck, Germany). Under a protective argon gas atmosphere, titanium powder grade 2 (supplier: Realizer $\mathrm{GmbH}$, Borchen, Ger- many) of average particle size of $60 \mu \mathrm{m}$ was evenly spread onto a titanium building platform after the platform has been lowered by $30 \mu \mathrm{m}$. A $200 \mathrm{~W}$ infrared laser then scanned over that powder bed. By the focused laser energy, the irradiated Ti particles fused with the underlying layer along the trajectories of the laser. This process was repeated layer-bylayer until the structures were completely produced. When the building platform and the implants had cooled down, they were removed from the building chamber and the implants were carefully detached from the underlying support structures, respectively, from the platform. As described in Ref., ${ }^{24}$ ultrasonic, chemical, and plasma cleaning procedures followed before the scaffolds were packed into a peel bag and gamma sterilized at $25 \mathrm{kGy}$.

The effective strut diameter $s$, the channel width $w$, and the surface morphology of the produced implants were determined by scanning electron microscopy (Hitachi TM3030, Japan,

Table 1. Structural Values of the Three Designed Series of Scaffolds with Engineered Orthogonal Lattice Geometry

\begin{tabular}{|c|c|c|c|c|c|c|c|c|c|c|c|}
\hline & $\begin{array}{l}\phi_{\mathrm{big}} \\
(\mathrm{mm})\end{array}$ & $\begin{array}{l}\emptyset_{\text {small }} \\
(\mathrm{mm})\end{array}$ & $\begin{array}{l}\mathrm{h}_{\text {total }} \\
(\mathrm{mm})\end{array}$ & $\begin{array}{c}\mathrm{s} \\
(\mathrm{mm})\end{array}$ & $\begin{array}{c}\mathrm{W} \\
(\mathrm{mm})\end{array}$ & $\begin{array}{l}\mathrm{V}_{\text {encl. }} \\
\left(\mathrm{mm}^{3}\right)\end{array}$ & $\begin{array}{c}\mathrm{V}_{\text {grid }} \\
\left(\mathrm{mm}^{3}\right)\end{array}$ & $\begin{array}{l}V_{\text {free }} \\
(\%)\end{array}$ & $\begin{array}{c}\mathrm{A}_{\text {grid }} \\
\left(\mathrm{mm}^{2}\right)\end{array}$ & $\begin{array}{c}\text { Transp } \\
(100)\end{array}$ & $\begin{array}{c}\text { Transp } \\
\text { (110) }\end{array}$ \\
\hline Series 1 & 7.5 & 6 & 4.2 & 0.2 & 0.3 & 154 & 59.41 & $61 \%$ & 983.5 & $36 \%$ & $12 \%$ \\
\hline Series 1 and 2 & 7.5 & 6 & 4.2 & 0.2 & 0.466 & 154 & 38.94 & $75 \%$ & 684.7 & $49 \%$ & $28 \%$ \\
\hline Series 1 and 3 & 7.5 & 6 & 4.2 & 0.2 & 0.8 & 154 & 20.93 & $86 \%$ & 390.1 & $64 \%$ & $48 \%$ \\
\hline Series 1 & 7.5 & 6 & 4.2 & 0.2 & 1.8 & 154 & 7.74 & $95 \%$ & 149.9 & $81 \%$ & $72 \%$ \\
\hline Series 2 & 7.5 & 6 & 4.2 & 0.5 & 0.466 & 156 & 90.96 & $42 \%$ & 566.2 & $23 \%$ & $0 \%$ \\
\hline Series 2 & 7.5 & 6 & 4.2 & 1.5 & 0.466 & 164 & 136.23 & $17 \%$ & 296.3 & $6 \%$ & $0 \%$ \\
\hline not tested & 7.5 & 6 & 4.2 & 1.7 & 0.3 & 166 & 144.76 & $13 \%$ & 281.7 & $2 \%$ & $0 \%$ \\
\hline not buildable & 7.5 & 6 & 3.7 & 0.1 & 0.8 & 135 & 6.46 & $95 \%$ & 245.5 & $79 \%$ & $69 \%$ \\
\hline Series 3 & 7.5 & 6 & 4.2 & 0.2 & 0.8 & 154 & 20.97 & $86 \%$ & 390.4 & $64 \%$ & $48 \%$ \\
\hline Series 3 & 7.5 & 6 & 4.7 & 0.3 & 0.8 & 173 & 41.25 & $76 \%$ & 480.9 & $53 \%$ & $33 \%$ \\
\hline Series 3 & 7.5 & 6 & 5.2 & 0.4 & 0.8 & 192 & 63.84 & $67 \%$ & 549.8 & $44 \%$ & $22 \%$ \\
\hline
\end{tabular}

Transp, transparency. 
$15 \mathrm{kV}$, BSE detector). The overall geometry was examined by vernier caliper (TESA Cal IP 67, TESA, Switzerland).

\section{Surgical procedure}

All animal experiments comply with the Animal Research: Reporting of In Vivo Experiments guidelines and were carried out in accordance with the EU Directive 2010/63/EU for animal experiments. Eighteen adult (12 months old) New Zealand White rabbits were used in this study. The animals' weight was between 3.5 and $4.0 \mathrm{~kg}$ and they were fed a standard laboratory diet. Animals were housed in groups of 2-4 and allocated to the four scaffolds applied per animal at random. The procedure was evaluated and accepted by the local authorities (108/2012 and 115/2015). To initiate the operation, the animals were anesthetized by an injection of $65 \mathrm{mg} / \mathrm{kg}$ ketamine and $4 \mathrm{mg} / \mathrm{kg}$ xylazine and maintained under anesthesia with isoflurane $/ \mathrm{O}_{2}$. After disinfection, an incision from the nasal bone to the midsagittal crest was made, the soft tissue deflected, and the periosteum removed. Next, four evenly distributed 6-mm-diameter craniotomy defects were prepared with a trephine bur under copious irrigation with sterile saline in the operation field. Then, all defects were completed with a rose burr $(1 \mathrm{~mm})$ to preserve the dura. Next, all the defects were flushed with saline to remove remaining debris and the implants were applied by press fitting. Each of the animals received four different treatment modalities. The treatment modalities were assigned at random for the first animal, and thereafter, cyclic permuted clockwise. The treatments were grouped for distance of rods and rod thickness. Each scaffold type was applied six times. After the completion of implant placement, the soft tissues were closed with interrupted sutures. Four weeks after operation, the rabbits were placed under general anesthesia and sacrificed by an overdose of pentobarbital. The cranium containing all four craniotomy sites was removed and placed in $40 \%$ ethanol.

\section{Embedding}

Embedding was performed as previously reported. ${ }^{24}$ In short, the specimens were prepared with a sequential water substitution process. It involved $48 \mathrm{~h}$ in $40 \%$ ethanol, $72 \mathrm{~h}$ in $70 \%$ ethanol (changed every $24 \mathrm{~h}$ ), $72 \mathrm{~h}$ in $96 \%$ ethanol, and finally, $72 \mathrm{~h}$ in $100 \%$ ethanol. Thereafter, samples were placed in xylene for $72 \mathrm{~h}$ (changed every $24 \mathrm{~h}$ ) followed by methyl methacrylate (MMA) for $72 \mathrm{~h}$ (Fluka 64200) and $100 \mathrm{~mL}$ MMA $+2 \mathrm{~g}$ dibenzoylperoxid (Fluka 38581 ) at $4^{\circ} \mathrm{C}$ for 4 days. For polymerization, samples were submerged in $100 \mathrm{~mL}$ MMA $+3 \mathrm{~g}$ dibenzoylperoxid $+10 \mathrm{~mL}$ plastoid $\mathrm{N}$ or dibutylphthalate (Merck 80019.25 ) at $37^{\circ} \mathrm{C}$ in an incubator. After embedding, the skull was cut into four pieces each containing one craniotomy site by using an EXAKT 300P saw (Exakt, Norderstedt, Germany). The samples were sectioned through the middle of the defect. Sections of $200 \mathrm{~mm}$ thickness were obtained, ground, and polished to a uniform thickness of $60-80 \mathrm{~mm}$. The specimens were surface stained with toluidine blue. ${ }^{46}$

\section{Histomorphometry}

Evaluation of all implants was performed from the middle section using image analysis software (Image-Pro Plus ${ }^{\circledR}$; Media Cybernetic, Silver Springs, MD). The area of interest
(AOI) was defined by the $6 \mathrm{~mm}$ defect dimension and the height of the implant. Corrections were made for differences in implant height in series 3 . We determined the area of new bone in the AOI as percentage of bone and bony integrated scaffold in the AOI (bony area, \%). For the empty control value, the average area occupied by the titanium implants of all designs was taken into account.

\section{Bone bridging}

The determination of bone bridging was performed as reported earlier. ${ }^{47,48}$ In brief, areas with bone tissue were projected onto the $x$-axis. Next, the stretches of the $x$-axis where bone formation had occurred at any level were summed up and related to the defect width of $6 \mathrm{~mm}$. Bone bridging is given in percentage of the defect width $(6 \mathrm{~mm})$ where bone formation had occurred.

\section{Statistical analysis}

The primary analysis unit was the animal. For all parameters tested, the treatment modalities were compared with a Kruskal-Wallis test, followed by pairwise comparison of treatment modalities with the Mann-Whitney test for dependent data (IBM SPSS v.19). $p$ values are displayed in the graphs and significance was set at a limit of $p<0.05$. Data from 18 rabbits are presented. Values are reported as mean \pm standard deviation.

\section{Results}

\section{Implant design and surface morphology}

The geometry of the final implants was verified microscopically and metrologically. The rod calibers $s$ and the channel width $w$ were determined in scanning electron images, whereas the total height and the diameters of the inner and outer cylindrical sections were determined by tactile gauge $(n=3)$. The lattice design and symmetry were absolutely reproduced in the physical scaffolds. Although the outer geometry of the additively manufactured implants $\left(h_{t o t}\right.$, $\phi_{\text {small }}$ and $\phi_{\text {big }}$ ) exactly corresponds to the designed geometry (see Table 1), the rod calibers $s$ trend to slightly oversize $5.9 \%$ in standard error of mean measurements in the top view and $12.8 \%$ in lateral view. This geometrical deviation mainly derives from powder particles that are sintered to the surface of the struts. The surface morphology, typical for SLM surfaces, shows an extensive decoration with fused titanium particles, which explains the increased strut size $s$. Consequently, the channel width $w$ of the as-printed structures tends to be reduced compared with the designed values: $1.2 \%$ in top view and $5.6 \%$ in lateral view.

\section{Titanium grid structures and bone regeneration}

All animals stayed in good health and in the ground sections, no signs of inflammation could be detected. Bone formation occurred close to and in contact with the bone substitutes irrespective of the designs used (Fig. 2), which indicates a good overall biocompatibility of all designs in all series.

\section{Rod distance in grid structures and bone regeneration}

In the first set of experiments, rod distances $w$ in the lattice structure were tested at a fixed rod caliber of $s=0.2 \mathrm{~mm}$. The histological sections (Fig. 3 ) revealed that based on the middle 


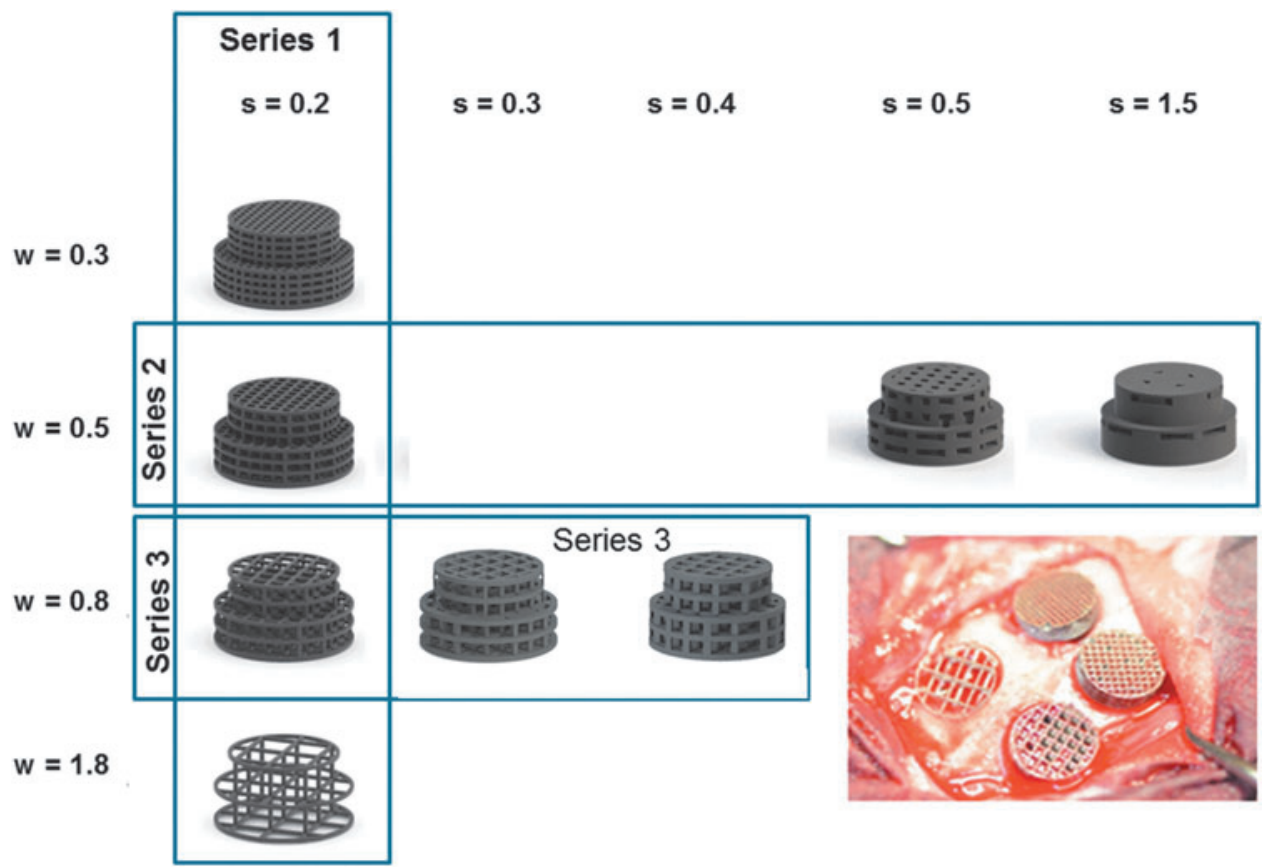

FIG. 2. Titanium grid designs. In a first series, different distances between rods were tested at a fixed rod caliber of $0.2 \mathrm{~mm}$. An intraoperative picture of the four designs after placement is provided in the right lower corner. The distances between the rods increased from 0.3 to $1.8 \mathrm{~mm}$. In the second series, different calibers of rods were tested ranging from 0.2 to $1.5 \mathrm{~mm}$ at a fixed rod distance of $0.5 \mathrm{~mm}$. In the third series, different calibers of rods were tested ranging from 0.2 to $0.4 \mathrm{~mm}$ at a fixed rod distance of $0.8 \mathrm{~mm}$.

sections, the microarchitecture variations in the distance between rods $w$ had a pronounced effect on bony regeneration of the defect. First, we determined bony regeneration of the defect in the middle sections. Here we found a significant relationship between rod distance $w$ and bone regeneration (Fig. 4a). At a rod distance of $w=0.3$ and $0.5 \mathrm{~mm}$, the percentage of bony regenerated area was $30.36 \% \pm 7.94 \%$ and $31.86 \% \pm 6.88 \%$, respectively. At a rod distance of $w=0.8 \mathrm{~mm}$, bony regenerated area was $51.98 \% \pm 13.10 \%$ and significantly higher than for the $0.5 \mathrm{~mm}$ distance $(p=0.005)$ and for the distance of $w=1.8 \mathrm{~mm}$ $(p=0.026)$. The percentage of bony regenerated area of the latter was $38.51 \pm 3.37$, and for the empty defect with an assumptive rod distance of $w=6 \mathrm{~mm}$ corresponding to the defect dimension, it was $14.75 \% \pm 5.25 \%$. For all implants with a rod distance between $w=0.3$ and $1.8 \mathrm{~mm}$, bony regenerated area was significantly higher $(p=0.003)$ than with the empty defect with an assumed rod distance of $w=6 \mathrm{~mm}$.

In this set of experiment, bony bridging was $57.50 \% \pm$ $24.57 \%$ for rod distance of $w=0.3 \mathrm{~mm}, 73.57 \% \pm 14.82 \%$ for rod distance of $w=0.5 \mathrm{~mm}, 90.83 \% \pm 16.22 \%$ for rod distance of $w=0.8 \mathrm{~mm}$, and $85.27 \% \pm 12.17 \%$ for a rod distance of $w=1.8 \mathrm{~mm}$ (Fig. 3b). A significant difference could be detected between rod distances of $w=0.3$ and $0.8 \mathrm{~mm}$. The empty defect with an assumed rod distance of $w=6 \mathrm{~mm}$, however, showed bony bridging to $37.77 \% \pm 22.20 \%$ and was significantly lower than all other groups in series $1(p=0.005)$.

\section{Rod dimension and bone regeneration}

In the series 2, we compared bone regeneration at a fixed rod distance of $0.5 \mathrm{~mm}$ for rod calibers of $0.2,0.5$, and $1.5 \mathrm{~mm}$ but no significant difference could be detected be- tween the implant groups based on bony regenerated area or defect bridging. Therefore, in the third series of experiments, we applied the aforementioned results about the optimal rod distance to be $w=0.8 \mathrm{~mm}$ and designed and produced grids with a constant distance between rods of $w=0.8 \mathrm{~mm}$ and varied the caliber of the rods from $s=0.2$ to $0.4 \mathrm{~mm}$. We failed to build an implant with a rod caliber of $s=0.1 \mathrm{~mm}$ due to procedural limitations of our manufacturing system. The histological sections (Fig. 5) revealed that based on the middle sections, the microarchitecture variations in rods caliber $s$ affected bony regeneration of the defect.

At a rod distance of $0.8 \mathrm{~mm}$, bone regeneration appeared to be significantly dependent on the rod calibers in the range of 0.2 to $0.4 \mathrm{~mm}$ (Fig. 6a). At a rod caliber of $s=0.2 \mathrm{~mm}$, percentage of bony regenerated area was $44.51 \% \pm 14.26 \%$. At a rod caliber of $s=0.3 \mathrm{~mm}$, bony regenerated area was $64.64 \% \pm 11.57 \%$ and significantly higher than for the $s=0.2 \mathrm{~mm}$ caliber $(p=0.035)$. No significant difference to 0.2 and $0.3 \mathrm{~mm}$ rod calibers $s$ was detected in comparison with the rod caliber of $0.4 \mathrm{~mm}$. The percentage of bony regenerated area of the latter was $57.63 \pm 11.73$, and for the empty defect it was $14.75 \% \pm 5.25 \%$. For all implants with a rod calibers between $s=0.2$ to $0.4 \mathrm{~mm}$, bony regenerated area was significantly higher $(p=0.003)$ than in empty defects with an assumed rod caliber of $0 \mathrm{~mm}$.

In this set of experiments, bony bridging was $67.62 \% \pm$ $24.50 \%$ for rod caliber of $r=0.2 \mathrm{~mm}, 79.616 \% \pm 18.15 \%$ for rod caliber of $r=0.3 \mathrm{~mm}$, and $71.90 \% \pm 14.66 \%$ for a rod caliber of $r=0.4 \mathrm{~mm}$ (Fig. 6b). No significant difference could be detected with calibers between 0.2 and $0.4 \mathrm{~mm}$. The empty defect with an assumed rod caliber of $0 \mathrm{~mm}$, however, showed bony bridging to $36.85 \% \pm 19.69 \%$ and was significantly lower than all other groups $(p=0.003)$. 
a

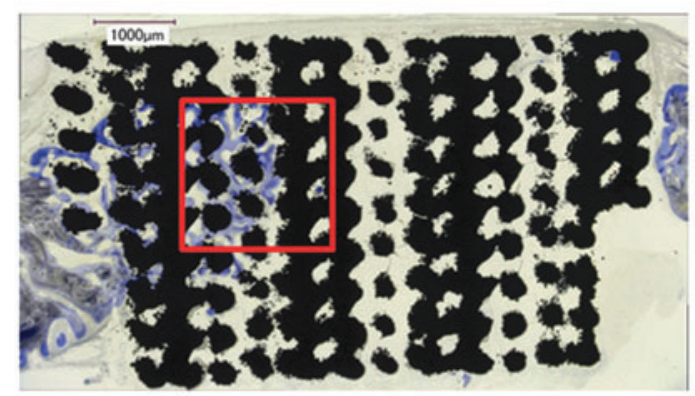

C

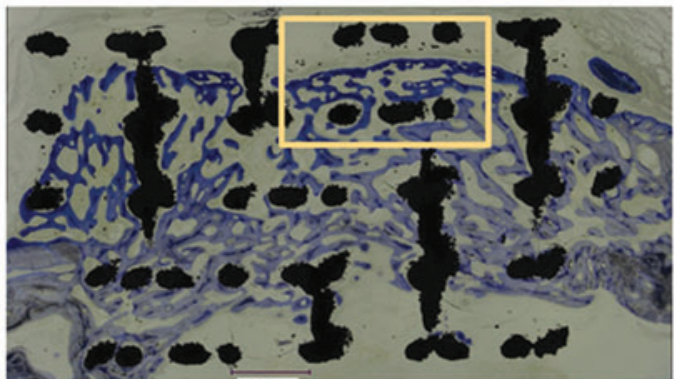

b

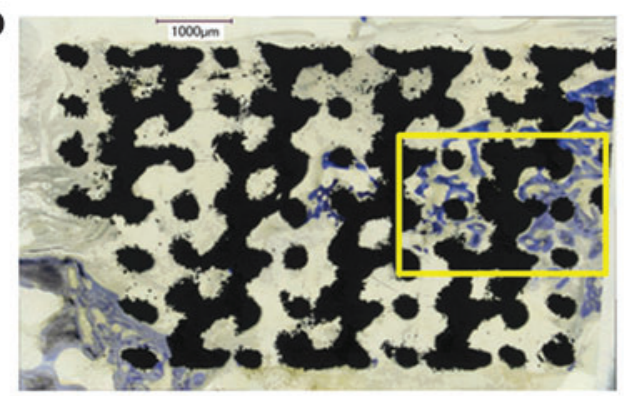

d

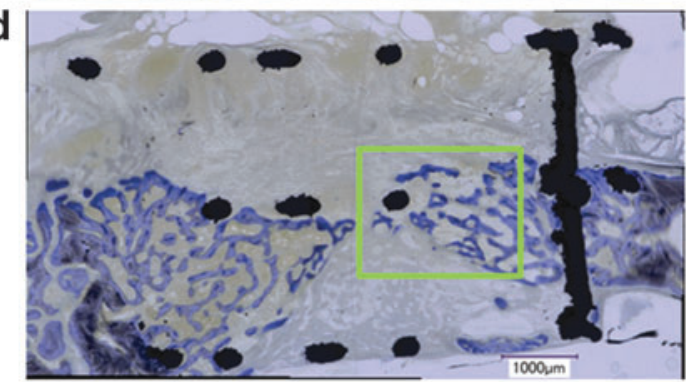

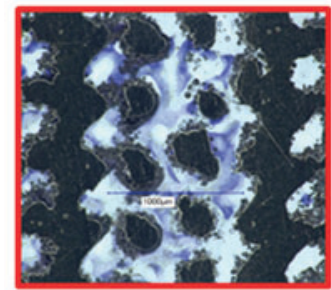
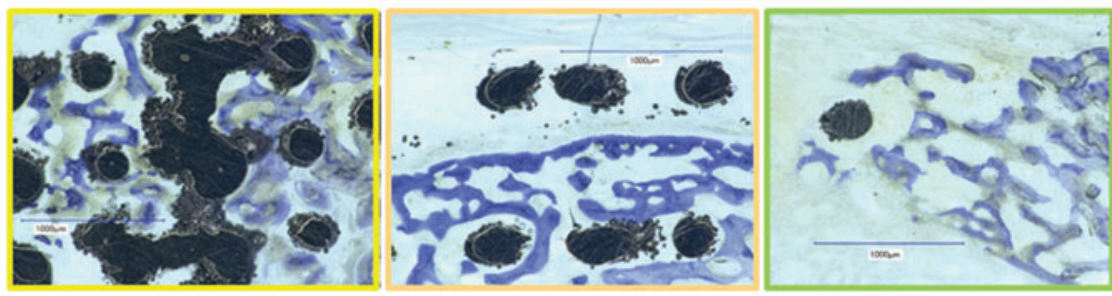

FIG. 3. Histological sections from the middle of the defect of series 1 at a fixed rod caliber of $s=0.2 \mathrm{~mm}$ and varying rod distances $w$. (a-d). For all scaffold designs varying in the distance between the rods, a histological section from 4 weeks postoperatively is shown. (a) Rod distance $w=0.3 \mathrm{~mm}$; (b) rod distance $w=0.5 \mathrm{~mm}$. (c) Rod distance $w=0.8 \mathrm{~mm}$, (d) rod distance $w=1.8 \mathrm{~mm}$. Scale bars indicate $1 \mathrm{~mm}$. Original magnifications were 100-fold. Bone appears as grayish-purple to purple, titanium as black. The projections appear unsymmetrical and do not reflect the orthogonal character of the struts because the cutting direction is slightly out of the lattices' main axis. In the lower panel, color-coded higher magnifications are provided for (a-d). The light blue-stained bone tissue is lamellar bone (also new, but later formed) on the initial woven bone structures stained dark blue and purple. In these 2.5 -fold higher magnifications, you can detect the lacunae from osteocytes.
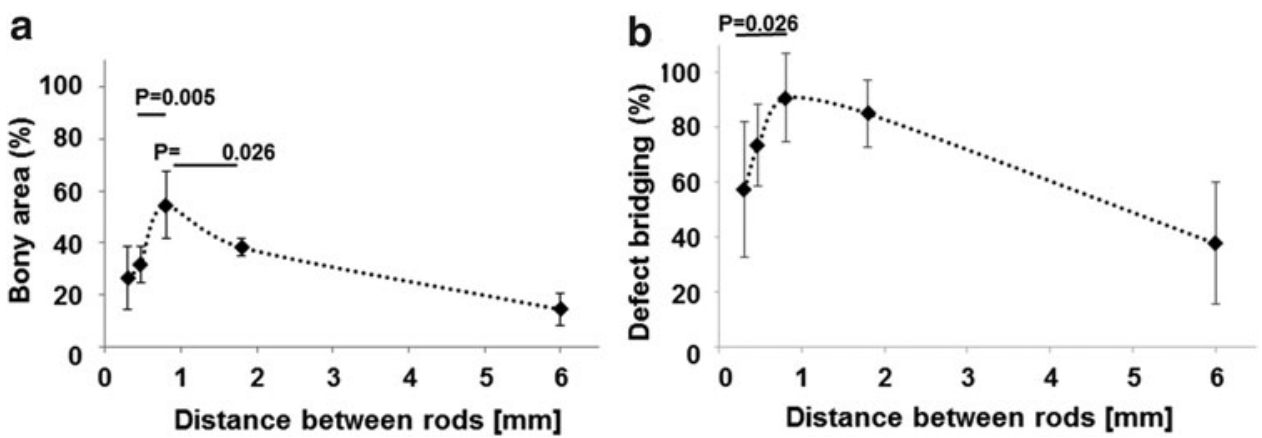

FIG. 4. Bone histomorphometric parameters in the AOI as a function of the rod distance $w$ in scaffolds with a constant rod caliber $s=0.2 \mathrm{~mm}$ from series 1 . (a) The formation of new bone in the presence of the tested implants is significantly elevated compared with empty defects with an assumed rod distance of $w=6 \mathrm{~mm}$. Comparison between the implants revealed that the implant with a rod distance of $w=0.8 \mathrm{~mm}$ performed significantly better than all other rod distances tested (b) Defect bridging was significantly increased in the presence of implants compared with untreated defects with an assumed rod distance of $w=6 \mathrm{~mm}$. Implants with a rod distance of $w=0.8 \mathrm{~mm}$ performed significantly better than with a rod distance of $w=0.3 \mathrm{~mm}$. Standard deviations are indicated. The level of significance is marked with a line between the corresponding samples. AOI, area of interest. 

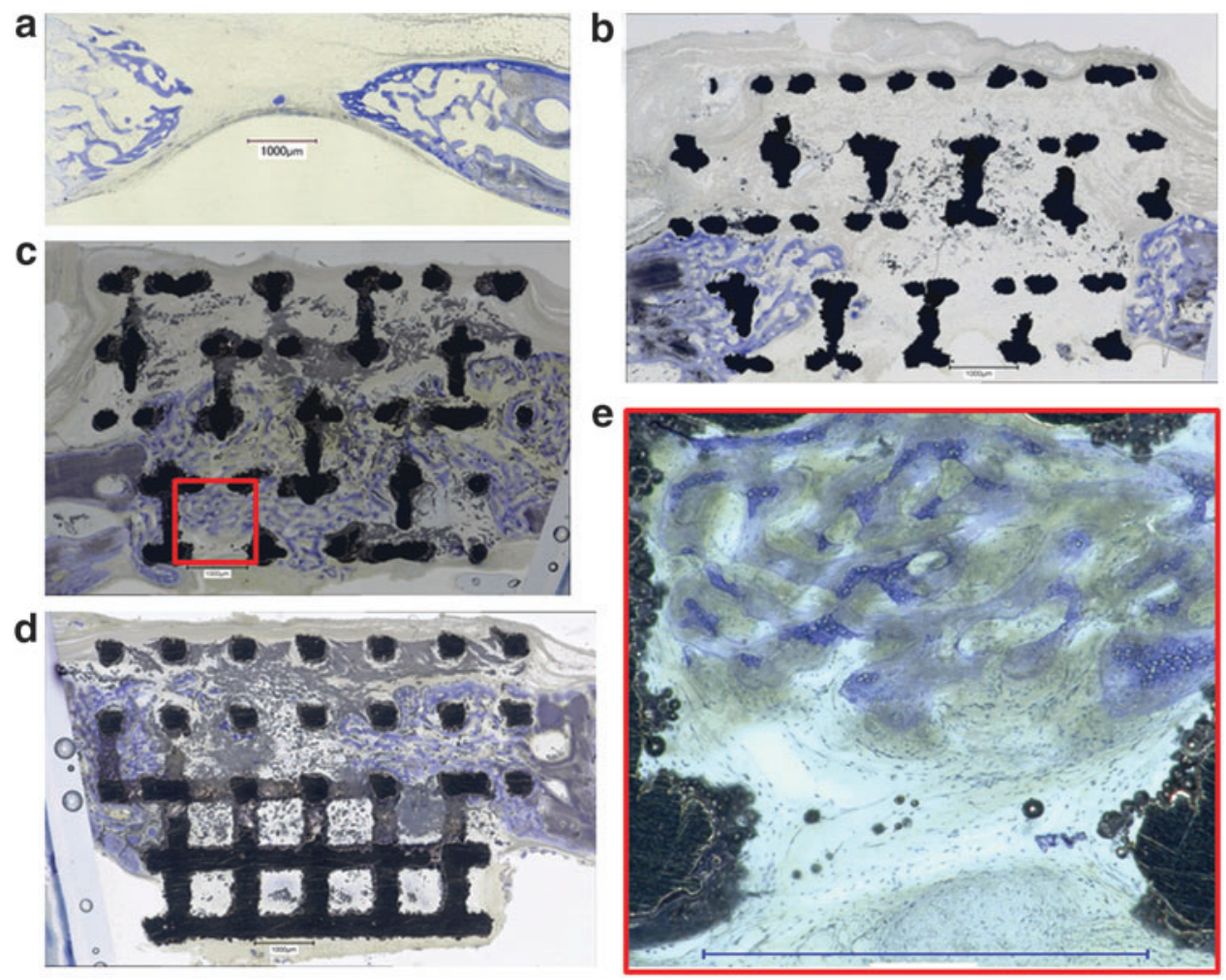

FIG. 5. Histological sections from the middle of the defect of series 3 at a fixed rod distance of $w=0.8 \mathrm{~mm}$ as a function of the rod caliber $s$. (a-d) For all scaffold designs varying in the rod caliber $s$, a histological section from 4 weeks postoperatively is displayed. (a) Empty defect with an assumed rod caliber of $s=0 \mathrm{~mm}$; (b) implant with rod caliber $s=0.2 \mathrm{~mm}$; (c) implant: rod caliber $0.3 \mathrm{~mm}$. (d) Implant with rod caliber $0.4 \mathrm{~mm}$. All scale bars indicate $1 \mathrm{~mm}$. Original magnifications were 100-fold. Bone appears as grayish-purple to purple, titanium as black. Some gray areas are artifacts from the embedding and grinding procedure and counted neither as bone nor as titanium. (e) Higher magnification from implant with a rod caliber of $0.3 \mathrm{~mm}$ (c). The corresponding area is indicated by a red box. The initial formed woven bone structures stained dark blue and purple. The light blue-stained bone tissue is newly formed lamellar bone, but later formed and deposited on the woven bone. In the sevenfold higher magnification, you can nicely see the lacunae from osteocytes.

\section{Discussion}

\section{SLM implant designs}

In previous studies, the influence of the surface morphology $^{24}$ and several microarchitectures ${ }^{25}$ were investigated.
Here, the lattice symmetry, surface morphology, the scaffolds bulk material, and the surface chemistry were kept constant while only lateral geometric parameters were varied: the strut calibers $s$ and the channel width $w$ or distance between the rods were adapted to study their effects on bone regeneration properties in vivo.
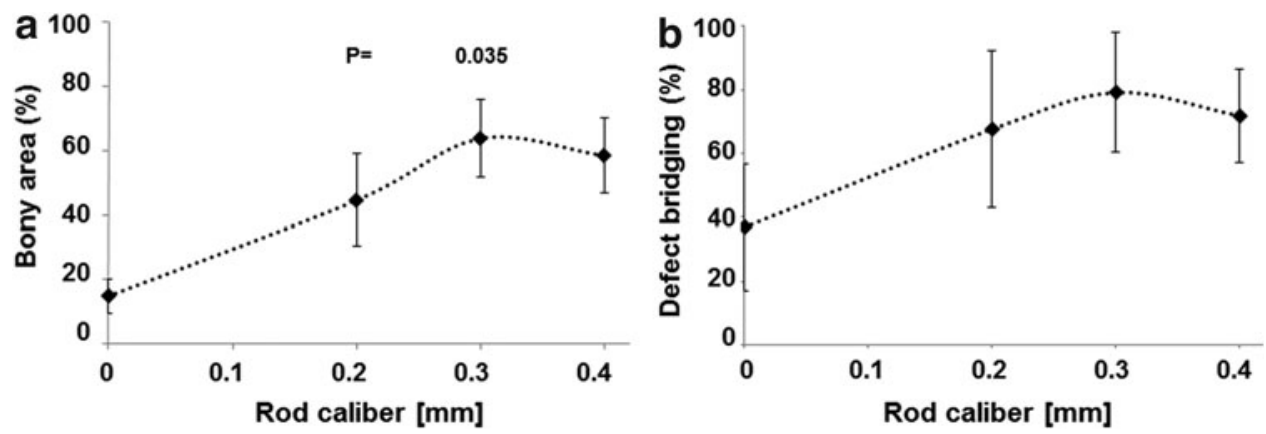

FIG. 6. Bony regeneration in the AOI of scaffolds with rod distances $w=0.8 \mathrm{~mm}$ and different rod calibers $s$. (a) The bony regenerated area for all tested implants is significantly higher than empty defects with an assumed rod diameter of 0 mm. Comparison between the implants revealed that the implant with a rod caliber of $s=0.3 \mathrm{~mm}$ performed significantly better than an implant with a rod caliber of $s=0.2 \mathrm{~mm}(p=0.035)$. No significant difference was seen with rod calibers of $0.4 \mathrm{~mm}$. Standard deviations are indicated. (b) Defect bridging was significantly increased in the presence of implants compared with untreated defects with an assumed rod caliber of $s=0 \mathrm{~mm}(p=0.003)$. Standard deviations are indicated. 


\section{Biological effects}

Lattice structures are often used as basic design principle for the microarchitecture of bone substitute scaffolds. ${ }^{26,49-51}$ Rod dimensions and distance between rods are in most cases selected based on mechanical needs to match them to bone ${ }^{51}$ or due to mechanical restrictions by the production process, for example, the mechanics of the extruded filament. ${ }^{19,52}$ Here we used titanium, known for its excellent osseointegration potential and AM by SLM to provide a high degree of design freedom to study bone regeneration in lattice structures in vivo dependent solely on the strut dimensions and the distance between struts. Our results show that bone regeneration and bony bridging facilitated by a titanium lattice structure are significantly improved when the distance between the rods is around $0.8 \mathrm{~mm}$ (Fig. 4) and the rod diameter is $0.3 \mathrm{~mm}$ (Fig. 6). Whereas the absolute thickness of the rods $s$ plays a minor role, the width $w$ between the struts appears as the most crucial factor.

Twenty years ago, the optimal pore size for bone tissue engineering was found to be between 0.3 and $0.5 \mathrm{~mm}^{20-22}$ and almost all porous bone substitutes up to now ${ }^{53}$ are designed accordingly. Based on our results on the distance between struts, the optimal pore matching this lattice would have a theoretical diameter of $0.89 \mathrm{~mm}$ and would be much bigger than previously proposed. That the optimal pore size in bone substitute exceeds $0.5 \mathrm{~mm}$ in diameter has recently been suggested by other researchers ${ }^{23}$ and us. ${ }^{25}$ Here we report on the optimal strut distance and strut caliber of lattice architectures.

Production-related limitations such as the averaged powder particle size of $60 \mu \mathrm{m}$, the laser focus of several tens of micrometers, and the powder layer height of $30 \mu \mathrm{m}$ lead to minimal, however, reproducible channel size $w$ of $0.3 \mathrm{~mm}$. Moreover, the size of the noncritical defect of $6 \mathrm{~mm}$ and the thickness of the calvarial bone of 2.8 to $3.5 \mathrm{~mm}$ are additional limitations of this study. Our results, however, show that strut distances of 0.5 and $1.8 \mathrm{~mm}$ are significantly less effective for bone regeneration than $0.8 \mathrm{~mm}$, and these structures are well within the inherent limitations of this model. From our previous work, ${ }^{25}$ we know that openings of up to $1.3 \mathrm{~mm}$ perform well in this model. That sets the optimal rod distances in a lattice structure from titanium to $0.8 \mathrm{~mm}$ and thus about two times further apart than the dogma for optimal porosity of bone substitutes suggests. ${ }^{32}$ Lattice structures with these characteristics can be produced by several AM methodologies and from diverse materials. However, further studies are needed to study the material dependency of the optimized microarchitecture in more detail.

Bone substitutes materials, even if they are osteoconductive, can support or hinder defect bridging. ${ }^{54}$ That is particularly true when open porous bone substitutes are compared with granular bone substitutes. ${ }^{55}$ Fast defect bridging appears to be facilitated by interconnected channels and lack of bottlenecks achieved perfectly by lattice microarchitectures. However, a minimum density of struts or an optimal distance between struts is needed, otherwise bony healing at low strut density (Fig. 3d) resembles bony healing in empty defects (Fig. 5a). Therefore, the struts at the optimal density provide the guiding cue to direct bone formation toward defect bridging, which is the initial goal of bone regeneration, since the major problems in bone regeneration are persistent delayed- or nonunions. ${ }^{56}$

\section{Conclusions}

In this systematic approach, identical bulk material, surface chemistry, surface topography, macrodesign, and lattice symmetry were used to study the influences of rod distance and rod caliber in a lattice titanium microarchitecture designed for bone regeneration purposes. The results show that significantly better results are achieved at a rod distance of $0.8 \mathrm{~mm}$ and a rod caliber of $0.3-0.4 \mathrm{~mm}$. Since AM and 3DP in many cases yield in a lattice microarchitecture, these results suggest to overcome the former dogma on channels of 0.3 to $0.5 \mathrm{~mm}$ and substitute them by channels of $0.8 \mathrm{~mm}$ for bone substitutes, where bone ingrowth has to occur fast and efficiently.

\section{Acknowledgments}

The authors thank Ana Perez and Alexandr Tchouboukov for excellent technical work and Thea Fleischmann for her support on the animal experiments. This research work was supported by grants from the Swiss National Science Foundation to F.E.W.

\section{Author Disclosure Statement}

No competing financial interests exist.

\section{References}

1. Hutmacher DW, Woodruff MA, Shakesheff K, et al. Direct fabrication as a patient-targeted therapeutic in a clinical environment. Methods Mol Biol 2012;868:327-340.

2. Langer RS, Vacanti JP. Tissue engineering: The challenges ahead. Sci Am 1999;280:86-89.

3. McHugh AJ. The role of polymer membrane formation in sustained release drug delivery systems. J Controlled Release 2005; 109:211-221.

4. Ehrbar M, Rizzi SC, Hlushchuk R, et al. Enzymatic formation of modular cell-instructive fibrin analogs for tissue engineering. Biomaterials 2007;28:3856-3866.

5. Lutolf MP, Weber FE, Schmoekel HG, et al. Repair of bone defects using synthetic mimetics of collagenous extracellular matrices. Nat Biotechnol 2003;21:513518.

6. Hutmacher DW, Sittinger M, Risbud MV. Scaffold-based tissue engineering: Rationale for computer-aided design and solid free-form fabrication systems. Trends Biotechnol 2004;22:354-362.

7. Mota C, Puppi D, Chiellini F, et al. Additive manufacturing techniques for the production of tissue engineering constructs. J Tissue Eng and Regen Med 2015;9:174-190.

8. ASTM. Standard Terminology for Additive ManufacturingGeneral Principles-Terminology. ASTM International, West Conshohocken, PA, 2015; DOI: 10.1520/ISOASTM5290015. www.astm.org 2015.

9. Woodruff MA, Lange C, Chen F, et al. Nano- to macroscale remodeling of functional tissue-engineered bone. Adv Healthc Mater 2013;2:546-551.

10. Kodama H. Automatic method for fabricating a threedimensional plastic model with photo-hardening polymer. Rev Sci Instrum 1981;52:1770.

11. Deckard CR. Method and apparatus for producing parts by selective laser sintering. US Patent No. 50177531989. 
12. Sachs E, Cima M, Cornie J. Three-dimensional printing: Rapid tooling and prototypes directly from a CAD model. CIRP Ann Manufactur Technol 1990;39:201-204.

13. Leukers B, Gülkan H, Irsen SH, et al. Hydroxyapatite scaffolds for bone tissue engineering made by $3 \mathrm{D}$ printing. J Mater Sci Mater Med 2005;16:1121-1124.

14. Crump SS. Apparatus und method for creating treedimensional objects. Patent No. 5121329, 1992.

15. Vozzi G, Flaim C, Ahluwalia A, et al. Fabrication of PLGA scaffolds using soft lithography and microsyringe deposition. Biomaterials 2003;24:2533-2540.

16. Vozzi G, Previti A, De Rossi D, et al. Microsyringe-based deposition of two-dimensional and three-dimensional polymer scaffolds with a well-defined geometry for application to tissue engineering. Tissue Eng 2002;8:1089-1098.

17. Hutmacher DW, Schantz T, Zein I, et al. Mechanical properties and cell cultural response of polycaprolactone scaffolds designed and fabricated via fused deposition modeling. J Biomed Mater Res 2001;55:203-216.

18. Schantz JT, Lim TC, Ning C, et al. Cranioplasty after trephination using a novel biodegradable burr hole cover: Technical case report. Neurosurgery 2006;58:ONS-E176.

19. Liu X, Rahaman MN, Liu Y, et al. Enhanced bone regeneration in rat calvarial defects implanted with surface-modified and BMP-loaded bioactive glass (13-93) scaffolds. Acta Biomater 2013;9:7506-7517.

20. Galois L, Mainard D. Bone ingrowth into two porous ceramics with different pore sizes: An experimental study. Acta Orthop Belg 2004;70:598-603.

21. Kuboki Y, Jin Q, Takita, H. Geometry of carriers controlling phenotypic expression in BMP-induced osteogenesis and chondrogenesis. J Bone Joint Surg 2001;83:S105-S115.

22. Murphy CM, Haugh MG, O'Brien FJ. The effect of mean pore size on cell attachment, proliferation and migration in collagen-glycosaminoglycan scaffolds for bone tissue engineering. Biomaterials 2010;31:461-466.

23. von Doernberg M-C, von Rechenberg B, Bohner M, et al. In vivo behavior of calcium phosphate scaffolds with four different pore sizes. Biomaterials 2006;27:5186-5198.

24. de Wild M, Schumacher R, Mayer K, et al. Bone regeneration by the osteoconductivity of porous titanium implants manufactured by selective laser melting: A histological and $\mu \mathrm{CT}$ study in the rabbit. Tissue Eng Part A 2013;19:2645-2654.

25. de Wild M, Zimmermann S, Rüegg J, et al. Influence of microarchitecture on osteoconduction and mechanics of porous titanium scaffolds generated by selective laser melting. J 3D Print Addit Manufactur 2016;3:142.

26. Boccaccio A, Uva AE, Fiorentino M, et al. Geometry design optimization of functionally graded scaffolds for bone tissue engineering: A mechanobiological approach. PLoS One 2016;11:e0146935.

27. Perez RA, Mestres G. Role of pore size and morphology in musculo-skeletal tissue regeneration. Mater Sci Eng C Mater Biol Appl 2016;61:922-939.

28. Gariboldi MI, Best SM. Effect of ceramic scaffold architectural parameters on biological response. Front Bioeng Biotechnol 2015;3:151.

29. Bružauskaitė I, Bironaite D, Bagdonas E, et al. Scaffolds and cells for tissue regeneration: Different scaffold pore sizesdifferent cell effects. Cytotechnology 2016;68:355-369.

30. Loh QL, Choong C. Three-dimensional scaffolds for tissue engineering applications: Role of porosity and pore size. Tissue Eng Part B Rev 2013;19:485-502.
31. Kim K, Yeatts A, Dean D, et al. Stereolithographic bone scaffold design parameters: osteogenic differentiation and signal expression. Tissue Eng Part B Rev 2010;16:523539.

32. Karageorgiou V, Kaplan D. Porosity of 3D biomaterial scaffolds and osteogenesis. Biomaterials 2005;26:54745491.

33. Imwinkelried T. Mechanical properties of open-pore titanium foam. J Biomed Mater Res A 2007;81:964-970.

34. Farré-Guasch E, Wolff J, Helder MN, et al. Application of additive manufacturing in oral and maxillofacial surgery. J Oral Maxillofac Surg 2015;73:2408-2418.

35. Gander T, Essig H, Metzler P, et al. Patient specific implants (PSI) in reconstruction of orbital floor and wall fractures. J CranioMaxillofac Surg 2015;43:126-130.

36. Wang G, Li J, Khadka A, et al. CAD/CAM and rapid prototyped titanium for reconstruction of ramus defect and condylar fracture caused by mandibular reduction. Oral Surgery Oral Med Oral Pathol Oral Radiol 2012;113:356361.

37. Stübinger S, Mosch I, Robotti $\mathrm{P}$, et al. Histological and biomechanical analysis of porous additive manufactured implants made by direct metal laser sintering: A pilot study in sheep. J Biomed Mater Res Part B Appl Biomater 2013;101:1154-1163.

38. Ma L, Zhou Y, Zhu Y, et al. 3D printed personalized titanium plates improve clinical outcome in microwave ablation of bone tumors around the knee. Sci Rep 2017; 7:7626.

39. Pobloth A-M, Checa S, Razi H, et al. Mechanobiologically optimized 3D titanium-mesh scaffolds enhance bone regeneration in critical segmental defects in sheep. Sci Transl Med 2018;10; pii: eaam8828: DOI: 10.1126/scitranslmed aam8828.

40. Wang D, Wang Y, Wu S, et al. Customized a Ti6Al4 V bone plate for complex pelvic fracture by selective laser melting. Materials 2017;10:35.

41. Cheng A, Cohen DJ, Kahn A, et al. Laser sintered porous Ti-6Al-4 V implants stimulate vertical bone growth. Ann Biomed Eng 2017;45:2025-2035.

42. Hollander DA, von Walter M, Wirtz T, et al. Structural, mechanical and in vitro characterization of individually structured Ti-6Al-4V produced by direct laser forming. Biomaterials 2006;27:955-963.

43. Hoffmann W, Bormann T, Rossi A, et al. Rapid prototyped porous NiTi scaffolds as bone substitutes. J Tissue Eng 2014;5: 2041731414540674.

44. Van Bael S, Chai YC, Truscello S, et al. The effect of pore geometry on the in vitro biological behavior of human periosteum-derived cells seeded on selective laser-melted Ti6Al4 V bone scaffolds. Acta Biomater 2012;8:28242834.

45. ASTM-F67. Standard Specification for Unalloyed Titanium, for Surgical Implant Applications (UNS R50250, UNS R50400, UNS R50550, UNS R50700). 2013.

46. Schenk RK, Olah AJ, Herrmann W. Preparation of calcified tissues for light microscopy. In: Dickson G.R., ed. Methods of Calcified Tissue Preparation.: Elsevire, Amsterdam, 1984; p. 1.

47. Kruse A, Jung RE, Nicholls F, et al. Bone regeneration in the presence of a synthetic hydroxyapatite/silica oxidebased and a xenogenic hydroxyapatite-based bone substitute material. Clin Oral Implants Res 2011;22:506-511. 
48. Schmidlin PR, Nicholls F, Kruse A, et al. Evaluation of moldable, in situ hardening calcium phosphate bone graft substitutes. Clin Oral Implants Res 2013;24:149157.

49. Do A-V, Khorsand B, Geary SM, et al. 3D printing of scaffolds for tissue regeneration applications. Adv Healthc Mater 2015;4:1742-1762.

50. Entezari A, Roohani-Esfahani S-I, Zhang Z, et al. Fracture behaviors of ceramic tissue scaffolds for load bearing applications. Sci Rep 2016;6:28816.

51. Roohani-Esfahani S-I, Newman P, Zreiqat H. Design and fabrication of 3D printed scaffolds with a mechanical strength comparable to cortical bone to repair large bone defects. Sci Rep 2016;6:19468.

52. Hutmacher DW. Scaffolds in tissue engineering bone and cartilage. Biomaterials 2000;21:2529-2543.

53. Qi X, Pei P, Zhu M, et al. Three dimensional printing of calcium sulfate and mesoporous bioactive glass scaffolds for improving bone regeneration in vitro and in vivo. Sci Rep 2017;7:42556.

54. Hong JY, Lee JS, Pang EK, et al. Impact of different synthetic bone fillers on healing of extraction sockets: An experimental study in dogs. Clin Oral Implants Res 2014; 25:e30-e37.

55. Karfeld-Sulzer LS, Ghayor C, Siegenthaler B, et al. Nmethyl pyrrolidone/bone morphogenetic protein-2 double delivery with in situ forming implants. J Control Release 2015;203:181-188.

56. Zura R, Xiong Z, Einhorn T, et al. Epidemiology of fracture nonunion in 18 human bones. JAMA Surg 2016;151: e162775.

Address correspondence to:

Franz E. Weber Oral Biotechnology and Bioengineering Center for Dental Medicine University of Zurich Plattenstrasse 11 Zürich 8032 Switzerland

E-mail: franz.weber@zzm.uzh.ch 